\title{
Primary cardiac tumour - An unusual incidental finding with significant implications
}

\author{
Tahir Nazir ${ }^{1,2 *}$, Shahid Tagari ${ }^{2}$, Yasser Abouemera ${ }^{2}$ and Irfan M Ahmed ${ }^{2}$ \\ ${ }^{1}$ Division of Cardiovascular Sciences, University of Manchester, Oxford Road, Manchester M13 9PL, UK \\ ${ }^{2}$ Department of Medicine, Lancashire Teaching Hospitals NHS Foundation Trust, Royal Preston Hospital, Preston PR2 9HT, UK
}

\begin{abstract}
Primary cardiac tumours are rarely seen in clinical practice and often present with symptoms like shortness of breath, syncope and palpitations [1]. None the less, even more rarely, cardiac tumours can be incidentally found on imaging intended for un-related clinical indications. These lesions carry a significant risk of serious complications even in asymptomatic individuals hence require urgent attention for further investigations and treatment. We describe a case of an otherwise fit and well woman who was found to have a left atrial mass on the CT scan intended to rule out a gastrointestinal malignancy. The left atrial mass was further evaluated by transthoracic echocardiogram and was considered to arise from the inter-atrial septum. She underwent a successful resection of this lesion and histological examination confirmed it to be a myxoma. We present a brief literature review on epidemiology, pathophysiology, clinical features, diagnosis and treatment options for cardiac myxomas. Any suspected primary cardiac mass should be further evaluated as a matter of urgency; surgery is usually the only effective treatment option available in most cases.
\end{abstract}

\section{Introduction}

Primary cardiac tumours are rare in clinical practice with an incidence ranging from $0.0017-0.19 \%$ in general population [2]. Due to the rarity of this condition, medical literature on pathophysiology, characterisation and treatment modalities is limited to case series and reports. We describe a case of incidentally discovered primary cardiac tumour and present a brief review on epidemiology, pathophysiology, clinical features, and diagnosis and treatment options.

\section{Case}

A 61 years old woman with no notable past medical history presented with per-rectal bleeding and weight loss. She denied any other symptoms including chest pain, shortness of breath, palpitations and syncope. Initial routine blood tests and ECG were within normal range. A CT scan of thorax, abdomen and pelvis was performed, mainly to rule out a GI malignancy. The scan showed an incidental $3.5 \times 3 \mathrm{~cm}$ large left atrial mass attached to the inter-atrial septum (IAS) (Figure 1). No extra cardiac lesions or lymphadenopathy were present on the scan. A transthoracic echocardiogram was carried out urgently that revealed a large mobile mass arising from IAS, abutting the mitral valve in systole (Figure 2). Systolic and diastolic functions of the heart were preserved. Cardiac catheterisation showed normal coronary arteries. Diagnosis of a primary cardiac tumour was made, and she was urgently referred to cardiothoracic centre for surgical resection. The tumour was excised in its entirety using a median sternotomy approach under general anaesthesia following which she made uneventful recovery. The excised mass was confirmed to be a myxoma on histology.

\section{Discussion}

In adults, over $75 \%$ of primary cardiac tumours are benign. Myxomas are the commonest cardiac tumours, followed by lipomas, fibromas, haemangiomas and neurofibromas. Highest incident of these

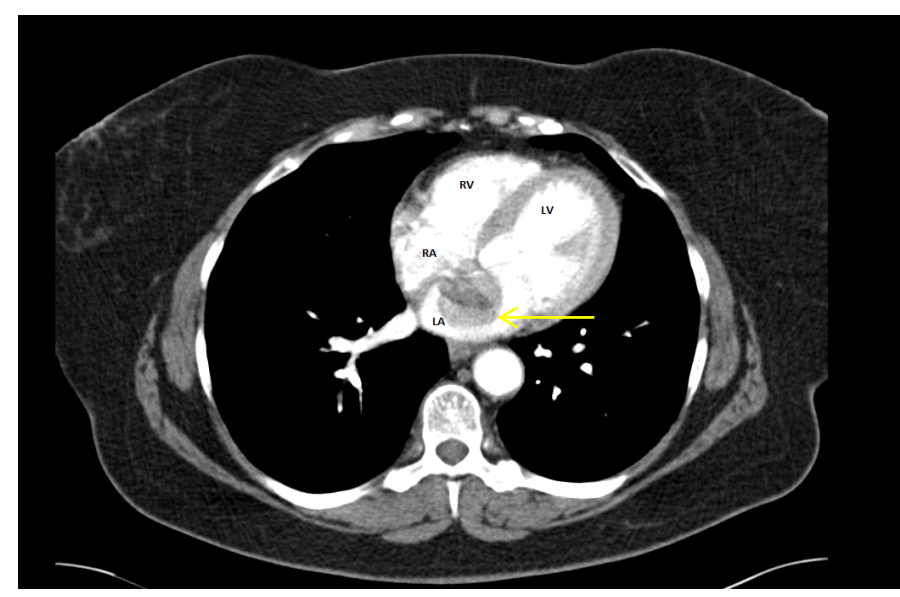

Figure 1. CT scan image demonstrating the presence of a large multi-lobular rounded shadow in the left atrium (arrow)

tumours is reported between third to sixth decade of life and most of these occur sporadically, although familial cases have been described in the literature too [3]. Majority of Myxomas arise from the inter-atrial septum and are found in the left atrium. The symptoms correlate poorly with the histological type of the tumour and mainly depend upon the size, consistency and the site of tumour. Clinical features can be broadly classified into three categories: embolization, intra-cardiac obstruction and constitutional features. Approximately, one third of the myxomas

*Correspondence to: Tahir Nazir, Division of Cardiovascular Sciences, University of Manchester, Oxford Road, Manchester M13 9PL, UK, E-mail: drtahirnazir@doctors.org.uk

Received: April 17, 2019; Accepted: April 26, 2019; Published: April 30, 2019 


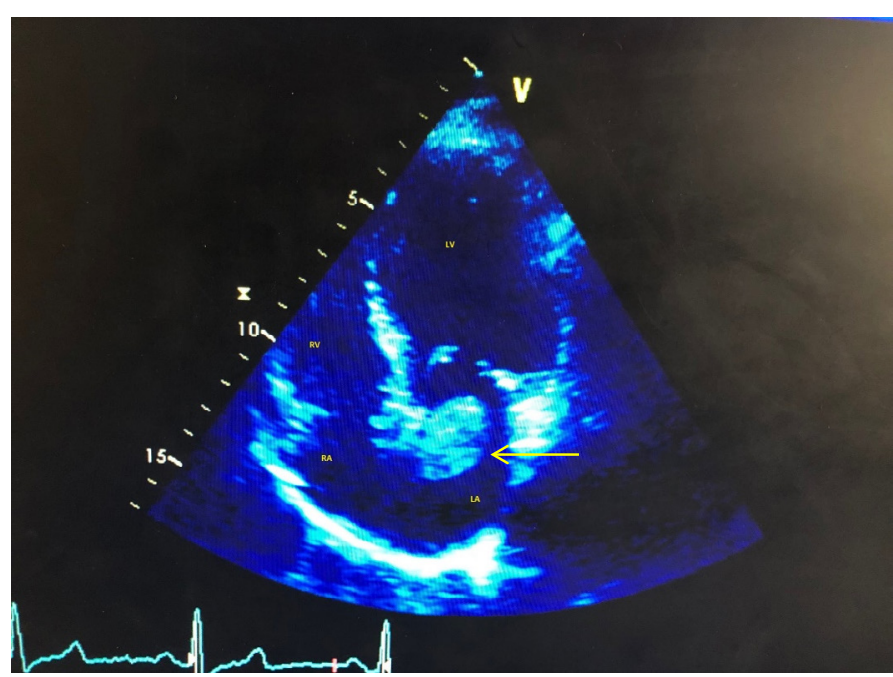

Figure 2. Transthoracic echo cardiogram (4 chamber view) showing a rounded mass in the left atrium originating from inter-atrial septum (arrow)

embolise leading to cerebral, renal and visceral infarctions; villous and papillary myxomas are friable and at a higher risk of embolization [4]. Dyspnoea, syncope and sudden cardiac death are the manifestations of intra-cardiac obstruction arising from these tumours. Transthoracic echocardiogram is a useful bedside investigation for initial assessment. Trans-oesophageal echocardiogram and cardiac MRI scan can help to further characterise these lesions by offering useful information about their consistency and anatomical relationship [5]. Coronary angiogram is reserved for those who are suspected to have concurrent coronary artery disease. Surgical resection in the only available effective treatment for primary cardiac tumours and should be performed promptly after the diagnosis is made in order to avoid complications [6].

\section{Conclusion}

Primary cardiac tumours are very rare and usually present with symptoms due to heart failure, embolization, arrhythmias and syncope. When found incidentally, they carry significant implications for further investigations and treatment. Untreated cardiac tumours can lead to serious complications including systemic embolization, stroke and sudden cardiac death.

\section{References}

1. Silverman NA (1980) Primary cardiac tumours. Ann Surg 191: 127-38. [Crossref]

2. Reynen K (1996) Frequency of primary tumours of the heart. Am J Cardiol 77: 107. [Crossref]

3. Mahilmaran A, Seshadri M, Nayar PG, Sudarsana G, Abraham KA (2003) Familial cardiac myxoma: Carney's complex. Texas Hear Inst J 30: 80-82. [Crossref]

4. Nath D, Arava S, Ray R, Bhoje AK, Saxena R, et al. (2017) Immunohistochemical characterization of glandular elements in glandular cardiac myxoma: Study of six cases. Indian J Pathol Microbiol 60: 319-323. [Crossref]

5. Abbas A, Garfath-Cox KAG, Brown IW, Shambrook JS, Peebles CR, et al. (2015) Cardiac MR assessment of cardiac myxomas. Br J Radiol 88: 20140599. [Crossref]

6. Lee KS, Kim GS, Jung Y, Jeong IS, Na KJ, et al. (2017) Surgical resection of cardiac myxoma - a 30-year single institutional experience. J Cardiothorac Surg 12: 18. [Crossref]

Copyright: (C2019 Nazir T. This is an open-access article distributed under the terms of the Creative Commons Attribution License, which permits unrestricted use, distribution, and reproduction in any medium, provided the original author and source are credited. 